\title{
Neue Therapieoption beim Colon irritabile
}

\begin{abstract}
Bei Patienten mit diarrhöbetontem Reizdarmsyndrom führt eine zweiwöchige Behandlung mit dem nicht resorbierbaren Antibiotikum Rifaximin zu einem signifikanten Rückgang der Symptomatik, insbesondere von Bauchschmerzen, der Entleerung von wässrigen Stühlen und Blähungen.
\end{abstract}

- Zumindest bei einem Teil der Patienten mit Reizdarmsyndrom dürfte die abnorme Zusammensetzung der Darmflora eine Rolle für die Entstehung der Symptomatik spielen. Daher hat man in zwei doppelblind geführten und placebokontrollierten Studien untersucht, ob eine Behandlung mit dreimal täglich $550 \mathrm{mg}$ Rifaximin, das bislang nur eine Zulassung für die Therapie der Reisediarrhö besitzt, zu einer Besserung der Symptome führen würde. Die Patienten wurden zwei Wochen lang behandelt und zehn Wochen lang nachbeobachtet, wobei die Symptome anhand einschlägiger Evaluationsskalen dokumentiert wurden.

Ausgewählt für die Studie wurden über 18-jährige Patienten, bei denen im Lauf der vorangegangenen zwei Jahre eine Koloskopie ohne Befund durchgeführt worden war und deren Diagnose

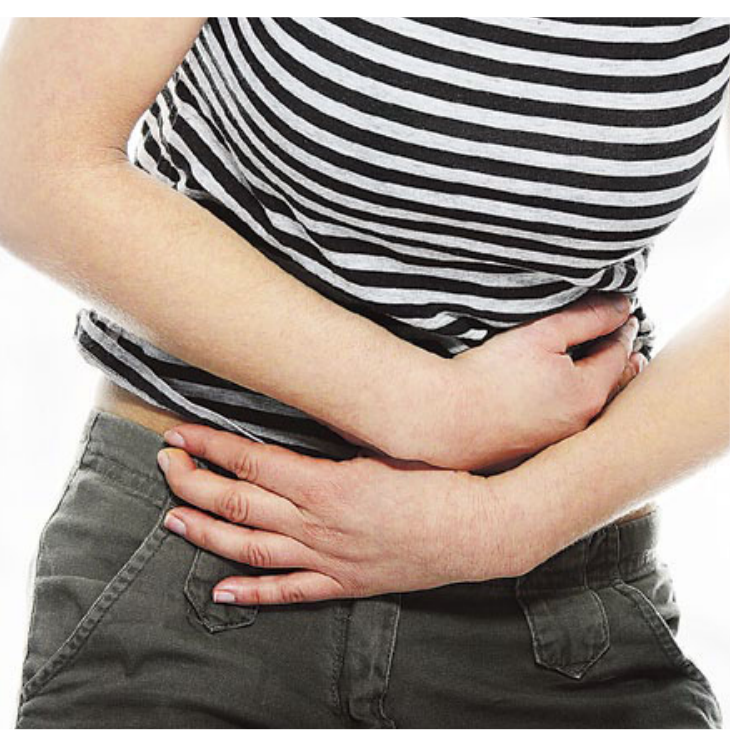

Colon irritabile: Hilft ein Antibiotikum?

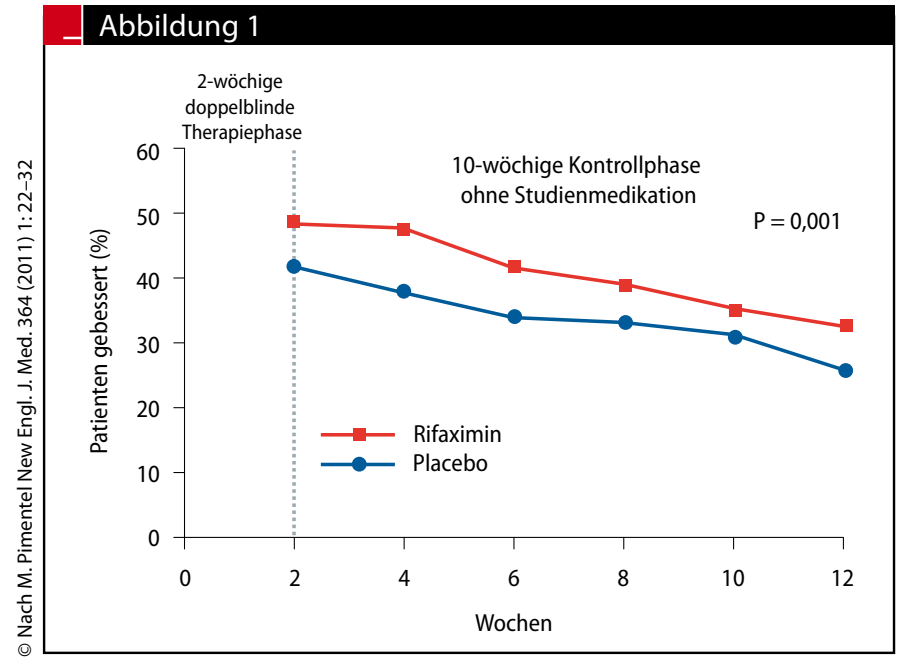

4 Abb. 1 Anteil von Patienten mit befriedigender Symptomenverbesserung anhand des Global IBS Symptomenscore unter Rifaximin und Placebo.

mittels der Rom-2-Kriterien gestellt wurde. Die Einnahme einschlägiger Medikamente wie Alosetron, Tegaserod, Lubiproston sowie Neuroleptika, Spasmolytika, Antidiarrhoika, Probiotika, und Narkotika war nicht gestattet. Antidepressiva vom Typ SSRI oder trizyklische Antidepressiva konnten dagegen weiter eingenommen werden, wenn die Dosis innerhalb der letzten sechs Wochen stabil war.

Insgesamt wurden 1260 Patienten mit Reizdarmsyndrom ohne Obstipation in die Studie eingeschlossen. Über 90\% der Patienten beendeten die Studie wie vorgesehen. In den ersten vier Wochen nach der Therapie mit Rifaximin kam es in der Verumgruppe bei 40,7\%, in der Placebogruppe dagegen nur bei $31,7 \%$ zu einer signifikanten Besserung der Symptomatik. Beim Symptom Blähungen betrug der Unterschied $40,2 \%$ vs. $30,3 \%$. Auch bei der gemeinsamen Bewertung der täglich dokumentierten Symptome des Colon irritabile wie Bauchschmerzen, Blähungen und Stuhlkonsistenz bemerkten signifikant mehr Patienten in der Rifaximingruppe eine Besserung, wobei sich der Unterschied über den gesamten Beobachtungszeitraum von zehn Wochen hielt (siehe Abb. 1).

\section{Kommentar}

Die Behandlung von Patienten mit Reizdarmsyndrom gestaltet sich meistens schwierig und ist von zahlreichen Enttäuschungen geprägt. Daher ist jede Erweiterung des therapeutischen Armamentariums durchaus willkommen. Dennoch erscheint die Gruppe der Patienten mit Reizdarmsyndrom zu heterogen, als dass mit einer Maßnahme für alle Patienten ein durchschlagender Effekt zu erwarten ist. Möglicherweise wäre dem antibiotischen Therapieansatz ein noch größerer Erfolg beschieden, wenn vorwiegend Patienten mit bakterieller Fehlbesiedelung behandelt würden. Leider besitzen wir derzeit noch keine wirklich guten prädiktiven differenzialtherapeutischen Parameter, um Patientengruppen zu identifizieren, bei denen entweder von Antibiotika, von Probiotika, von Prokinetika oder Psychopharmaka der größte Effekt zu erwarten ist. Immerhin hat man in der Studie eine gewisse Einschränkung dadurch getroffen, dass nur Patienten mit vorwiegender Neigung zu Durchfällen, nicht jedoch Obstipation einbezogen wurden. Wie beeinflussbar die Symptomatik von Patienten mit Reizdarmsyndrom allein durch eine offensichtlich intensive Studienbetreuung ist, zeigt der Umstand, dass auch die Patienten der Placebogruppe sich im Lauf der dreimonatigen Studie deutlich gebessert haben.

H. S. FÜESSL *

- M. Pimentel et al.

(Korres.: Dr. Forbes, 1700 Perimeter Park Dr, Morrisville, NC 27560): Rifaximin therapy for patients with irritable bowel syndrome without constipation. New Engl J Med 364 (2011) 1: 22-32 